\title{
Dynamical Properties of BOLD Activity from the Ventral Posteromedial Cortex Associated with Meditation and Attentional Skills
}

\author{
Giuseppe Pagnoni \\ Department of Biomedical Sciences, University of Modena and Reggio Emilia, I-41125 Modena, Italy
}

Neuroimaging data suggest a link between the spontaneous production of thoughts during wakeful rest and slow fluctuations of activity in the default mode network (DMN), a set of brain regions with high basal metabolism and a major neural hub in the ventral posteromedial cortex (vPMC). Meta-awareness and regulation of mind-wandering are core cognitive components of most contemplative practices and to study their impact on DMN activity, we collected functional MRI (fMRI) data from a cohort of experienced Zen meditators and meditation-naive controls engaging in a basic attention-to-breathing protocol. We observed a significant group difference in the skewness of the fMRI BOLD signal from the vPMC, suggesting that the relative incidence of states of elevated vPMC activity was lower in meditators; furthermore, the same parameter was significantly correlated with performance on a rapid visual information processing (RVIP) test for sustained attention conducted outside the scanner. Finally, a functional connectivity analysis with the vPMC seed revealed a significant association of RVIP performance with the degree of temporal correlation between VPMC and the right temporoparietal junction (TPJ), a region strongly implicated in stimulus-triggered reorienting of attention. Together, these findings suggest that the vPMC BOLD signal skewness and the temporal relationship of vPMC and TPJ activities reflect the dynamic tension between mind-wandering, meta-awareness, and directed attention, and may represent a useful endophenotype for studying individual differences in attentional abilities and the impairment of the latter in specific clinical conditions.

\section{Introduction}

A sizeable share of human neuroimaging research in the last decade has been directed at the so-called "resting state," a loosely defined experimental condition of unconstrained mental activity where subjects are instructed to remain passive and try not to engage in any specific motor or mental act. The discovery of a consistent set of brain regions with higher metabolism at rest than during externally directed tasks (Shulman et al., 1997) has sparked considerable interest in the functional significance of such a "default mode network" (DMN) (Raichle et al., 2001). Substantial evidence has implicated the DMN in the spontaneous thought processes arising in the absence of challenging external demands (Binder et al., 1999; McKiernan et al., 2006; Mason et al., 2007; Christoff et al., 2009), processes characterized by important verbal and semantic (Binder et al., 2008; Wirth et al., 2011), mnestic (Christoff et al., 2004; Vincent et al., 2006), scene construction

Received Aug. 11, 2011; revised Feb. 13, 2012; accepted Feb. 27, 2012.

Author contributions: G.P. designed research; G.P. performed research; G.P. analyzed data; G.P. wrote the paper. This work was supported by the Emory Center for Research on Complementary and Alternative Medicine in Neurodegenerative Diseases (National Institute of Health Grant P30-AT00609). The funders had no role in study design, data collection and analysis, decision to publish, or preparation of the manuscript. The author thanks $R$. Cameron Craddock, Daniel F. Drake, and Milos Cekic for the many helpful discussions.

The author has no competing financial interests.

Correspondence should be addressed to Giuseppe Pagnoni, Dip. Scienze Biomediche, Sezione Fisiologia, Università di Modena e Reggio Emilia, Via Campi 287, I-41125 Modena, Italy. E-mail: giuseppe.pagnoni@unimore.it.

DOI:10.1523/JNEUROSCI.4135-11.2012

Copyright $\odot 2012$ the authors $\quad 0270-6474 / 12 / 325242-08 \$ 15.00 / 0$
(Hassabis and Maguire, 2007), and mental-simulation components (Buckner and Carroll, 2007).

The relationship between directed attention and taskunrelated thoughts is a key pragmatic interest of many contemplative practices (Lutz et al., 2008). Within the Buddhist tradition in particular, an enhanced capacity of attentional regulation and awareness of the subtle movements of the mind is considered instrumental in recognizing, and thus emancipating oneself from, unwholesome mental habits, while activating at the same time a cognitive deconstruction of the emotional components of afflictive states that is held to be conducive to greater well being (Mizuno, 1996).

In the present study, we examined the fluctuations of brain activity associated with a self-regulated attention-to-breathing meditative task in a sample of regular Zen practitioners and matched control subjects, using functional magnetic resonance imaging (fMRI). We focused on the ventral posteromedial cortex (vPMC), a core region of the DMN (Fransson and Marrelec, 2008; Hagmann et al., 2008), including posterior cingulate and retrosplenial cortices, which showed increased response to conceptual processing in the same batch of subjects during the performance of a lexical-decision task (Pagnoni et al., 2008).

Under the working hypothesis that spontaneous DMN activation represents a source of internal noise affecting directed attention (Sonuga-Barke and Castellanos, 2007), we estimated the relative incidence of states of elevated vPMC activity by computing the skewness of its fMRI time course. Since a signal with negative skewness is characterized by a relatively greater repre- 
sentation of states above the signal's mean level, we predicted that (1) the vPMC signal would exhibit a less negative (or more positive) skewness in meditators compared to controls, and (2) subjects whose vPMC signal was more negatively skewed would be more prone to distraction and thus would score more poorly on a rapid visual information processing (RVIP) task designed to probe sustained attention and working memory (Wesnes and Warburton, 1983). To further characterize the relationship between vPMC activity and sustained attention, we also performed a functional connectivity (fc) analysis with the vPMC seed, examining how the network of brain regions temporally linked to vPMC during attention-to-breathing varied with individual RVIP performance.

\section{Materials and Methods}

Subjects. Twelve volunteers with $>3$ years of daily practice of Zen meditation (MEDT group) were recruited from the local community and meditation centers, along with 12 control subjects (CTRL group) who never practiced meditation before. The groups were matched by gender (MEDT, 10 males; CTRL, 9 males), age (mean \pm SD: MEDT, $37.3 \pm 7.2$ years; CTRL, $35.3 \pm 5.9$ years; two-tailed two-sample $t$ test: $p=0.45$ ), and education level (mean \pm SD: MEDT, $17.8 \pm 2.5$ years; CTRL, $17.6 \pm$ 1.6 years; $p=0.85$ ). All participants were native speakers of English and right-handed, except one meditator who was ambidextrous. Participants gave written informed consent for a protocol approved by the Emory University Institutional Review Board.

Rapid visual information processing test. Individual capacity for sustained attention was assessed $\sim 1$ week before the MRI scanning session via a RVIP test from the computerized neuropsychological battery CANTAB (Sahakian and Owen, 1992). The test requires the continuous monitoring of a stream of single digits, appearing rapidly in pseudorandom order in the center of a computer screen, for the occurrence of three specific target sequences (2-4-6, 3-5-7, 4-6-8). Stimuli (stim) are presented at a rate of $100 \mathrm{stim} / \mathrm{min}$, with 32 target sequences overall and a total duration of $4 \mathrm{~min}$. Performance is computed in terms of response times (RT) and $A^{\prime}$, a nonparametric version of signal detection theory's sensitivity index $d^{\prime}$, estimating the ability to detect targets on a scale from 0 to 1 ( 1 representing perfect performance) based on the number of hits and false alarms (Green and Swets, 1966). Each subject performed the task twice, with an interval of $\sim 30 \mathrm{~min}$ between repetitions, as part of a different section of the study examining the relationship of autonomic function during meditation and attentional performance (to be discussed elsewhere). Since individual scores exhibited a good test-retest Pearson's correlation $\left(A^{\prime}: r=0.78, p=0.000008\right.$; RT: $r=$ $0.66, p=0.0004)$, and a paired $t$ test for differences between the two sessions was nonsignificant $\left(A^{\prime}: p=0.59\right.$; RT: $\left.p=0.43\right)$, we selected the average of the measurements at the two time points as a more stable index of the individual capacity for sustained attention.

MRI scanning protocol and instructions to subjects. Scanning was performed with a 3.0 tesla Siemens Magnetom Trio scanner. The imaging session began with the acquisition of a T1-weighted high-resolution anatomical image (MPRAGE, 176 sagittal slices, voxel size: $1 \times 1 \times 1 \mathrm{~mm}$ ), followed by an echo-planar imaging (EPI) scan (gradient echo, 520 volumes, 35 axial slices, voxel size: $3 \times 3 \times 3 \mathrm{~mm}, \mathrm{TR}=2.35 \mathrm{~s}$, TE $=30 \mathrm{~ms}$ ), during which the volunteers engaged in a lexical-decision task, details and findings of which are reported in Pagnoni et al. (2008). The functional imaging run that is the focus of the present report was performed last (gradient-echo EPI, 200 volumes, 35 axial slices, voxel size: $3 \times 3 \times 3$ $\mathrm{mm}, \mathrm{TR}=2.35 \mathrm{~s}, \mathrm{TE}=30 \mathrm{~ms})$. Participants were instructed to keep their eyes open and pay attention to their breathing throughout the full length of the run $(\sim 8 \mathrm{~min})$, and to calmly return their attention to breathing every time they found themselves distracted by thoughts, memories, or physical sensations; a fixation cross was kept on the MRI display screen to help concentration and minimize eye movement. Respiration and heart rates were also acquired during the scans via a nasal cannula and an oxymeter, although, due to technical issues, we were able to get usable data for only a subset of the 24 subjects (respiration rate: 10 subjects, 4
CTRL + 6 MEDT; heart rate: 19 subjects, 10 CTRL + 9 MEDT). An eye-tracker camera, providing a real-time image of the subject's eye on a computer screen in the MRI console room, was also used to visually monitor potential episodes of drowsiness during the scans; the collection of eye-tracking data of sufficient quality for quantitative analysis, however, proved to be too challenging with the available set up and in the allotted scan time, and was not further pursued after the first few trial sessions.

Image preprocessing and analysis. Image processing was performed with the software packages AFNI (Cox, 1996) and SPM5 (http://www.fil. ion.ucl.ac.uk/spm); additional statistical analyses and graphs were obtained with the software R (R Development Core Team, 2011).

Basic preprocessing. For each subject, the EPI scans were corrected for differences in slice acquisition times and head motion; the T1-weighted image was spatially registered to the mean EPI image with a rigid-body transformation and subsequently warped to the Montreal Neurological Institute (MNI) brain template using the unified segmentation approach (Ashburner and Friston, 2005) in SPM5. The estimated warping parameters were applied to the EPI volumes to bring all subjects' data into standard space.

Extraction of global tissue-specific BOLD signals. The segmentation of the $\mathrm{T} 1$-weighted image produced individual tissue probability maps (TPMs) in native space for gray matter (GM), white matter (WM), and CSF. The TPMs were resampled to match the EPI resolution and converted to binary masks (threshold: $p=0.2$ ); the WM and CSF masks were also eroded by 1 voxel to minimize contamination from gray matter signal by partial volume effect, and the CSF mask was further manually edited to include only voxels from the lateral ventricles, since voxels close to the pial surface have been shown to contain strong gray matter components (Jo et al., 2010). The representative BOLD signals from the GM, the white matter-eroded (WMe), and the lateral ventricles-eroded (LVe) masks were then extracted as within-mask averages of the motioncorrected EPI time series in native space.

Removal of nuisance covariates and temporal filtering. Voxelwise removal by linear regression of the whole-brain average BOLD time course [global signal (GS)] is a common preprocessing step used by many fc studies to minimize the influence of physiological confounds of nonneural origin, such as cardiac and respiratory aliased components (Birn et al., 2006, 2008; Chang and Glover, 2009), on the local BOLD signal fluctuations. Although GS regression has been shown to mathematically mandate the appearance of anticorrelations (Murphy et al., 2009), it also seems to improve significantly both the specificity of detected functional connectivity networks and their match to known structural connectivity pathways (Fox et al., 2009; Weissenbacher et al., 2009). Furthermore, recent evidence of a spatially widespread neural signal contributing to the BOLD GS (Schölvinck et al., 2010) suggests that the latter may need to be accounted for to unmask finer temporal relationships between the activities of different cortical areas or specific temporal patterns of local activity.

Based on the above considerations and aiming to maximize the sensitivity of our analyses, we linearly regressed out from the EPI time series three global covariates, corresponding to the average BOLD signals from the WMe, LVe, and GM masks. Splitting the global signal into components of likely nonneural (WMe and LVe) and neural (GM) origin allowed us to assess the specific impact of the latter component on the obtained results (see Functional connectivity analysis, below). The preprocessed EPI time series were spatially and temporally filtered according to the following steps, implemented by the AFNI routine 3dBandpass: (1) a constant plus a linear and a quadratic trend were removed from each voxel's time series; (2) the time series were bandpass-filtered in the 0.01$0.1 \mathrm{~Hz}$ range; (3) the head motion parameters estimated during motion correction, along with the average BOLD time courses from the WMe, $\mathrm{LVe}$, and GM masks, were similarly bandpass-filtered and least-squares removed from the filtered EPI time series; and (4) the EPI volumes were spatially smoothed with a $6 \mathrm{~mm}$ FWHM Gaussian kernel within an inbrain mask only.

Definition of $v$ PMC ROI and computation of BOLD signal skewness. The vPMC ROI was defined as the posteromedial cortex activation cluster from the word versus nonword contrast in the group analysis of the lexical decision task performed by each subject in the same MRI session 
A
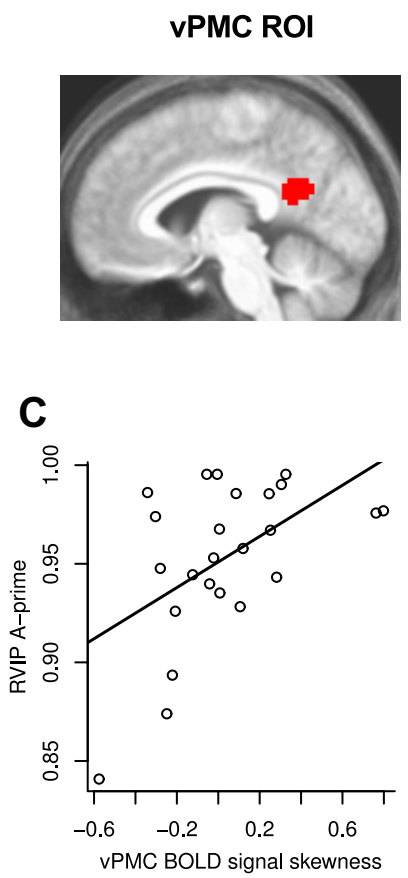

B

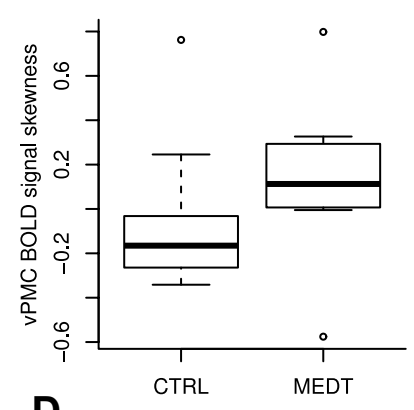

D

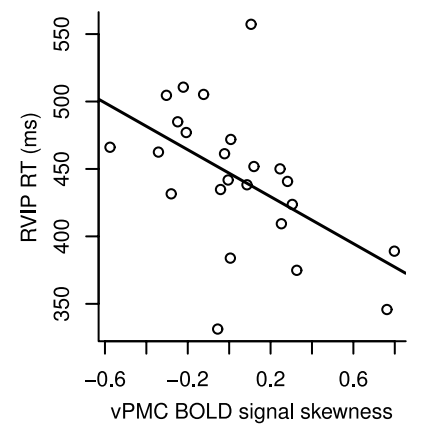

Figure 1. A, Depiction of the vPMC cluster obtained from the group analysis of the lexicaldecision task described by Pagnoni et al. (2008) and used in this study as a region of interest. $\boldsymbol{B}$, Box-and-whisker plot of the distribution of the VPMC BOLD signal skewness values in meditators (MEDT) and control subjects (CTRL), where outlier subjects (skewness values farther than 1.5 times the interquartile range from the box edges) are plotted as individual circles. $C, D$, Scatterplots and linear fits of the individual RVIP $A^{\prime}$ and RT scores, respectively, versus the VPMC BOLD signal skewness values.

and reported in Pagnoni et al. (2008). The MNI coordinates of the vPMC cluster's center of mass were $-6,-56,22$, and the cluster size was 82 voxels $\left(2214 \mathrm{~mm}^{3}\right)$ (Fig. $1 \mathrm{~A}$ ). We selected the vPMC as a single bestrepresentative time course for the evaluation of the temporal characteristics of DMN activity because it is the typical seed location used in functional connectivity studies to detect the DMN (Greicius et al., 2003) and therefore a region that is warranted to share an important portion of the dynamic behavior of all DMN components. Moreover, the posteromedial cortex was recently identified as a main cortical hub in terms of its structural and functional connectivity (Fransson and Marrelec, 2008; Hagmann et al., 2008), making it a strong candidate for modulating the interaction of large-scale neural systems such as the DMN and frontoparietal attentional circuits.

The average filtered BOLD time series from the vPMC ROI, $y(t)=y_{1}$, $y_{2}, \ldots, y_{200}$, was extracted for every subject and its skewness computed as $\sum_{i=1}^{200}\left(y_{i}-\mu\right)^{3} / \sigma^{3}$, where $\mu$ indicates the time series' mean and $\sigma$ its standard deviation (Joanes and Gill, 1998). To test the hypothesis of meditators showing a less negative (or more positive) skewness of the vPMC BOLD signal compared with controls, we performed a nonparametric Wilcoxon rank sum test for group differences. To test the hypothesis of less negative/more positive vPMC BOLD signal's skewness values being associated with better RVIP scores $\left(A^{\prime}\right.$ and RT), we performed Pearson's correlation tests between these quantities across all subjects.

Additionally, as a post hoc verification of the specificity of the effects of interest to the $\mathrm{VPMC}$, we computed the BOLD signal skewness and performed the analyses described above for the other regions of the DMN activated by semantic processing in the lexical-decision task (for coordinates and spatial extent, see Table 1) (for further details, see Pagnoni et al., 2008). For the same purposes, we also conducted an exploratory voxelwise correlation analysis of BOLD skewness values and individual RVIP $A^{\prime}$ scores across the whole brain.

Since the lexical-decision task always preceded the attention-tobreathing run in the MRI scanning session, neural hysteresis effects

Table 1. Additional R0Is from the words-versus-nonword contrast in the lexicaldecision task

\begin{tabular}{lllrr}
\hline & & \multicolumn{3}{c}{ Center-of-mass MNI coordinates } \\
\cline { 3 - 5 } Region & Size (in voxels) & $x$ & \multicolumn{1}{c}{$y$} & \multicolumn{1}{c}{$z$} \\
\hline L MCC & 195 & -10 & -34 & 47 \\
L sup frontal g (1) & 146 & -22 & 30 & 51 \\
L sup frontal g (2) & 44 & -15 & 59 & 18 \\
L angular g (1) & 145 & -33 & -76 & 38 \\
L angular g (2) & 69 & -51 & -61 & 22 \\
L rACC & 46 & -4 & 45 & -10 \\
L inf temporal g & 27 & -63 & -23 & -20 \\
\hline
\end{tabular}

Numbers in parentheses index different clusters in the same anatomical region. $L$, left; sup, superior; inf, inferior; $g$, gyrus; MCC, middle cingulate cortex; rACC, rostral anterior cingulate cortex.

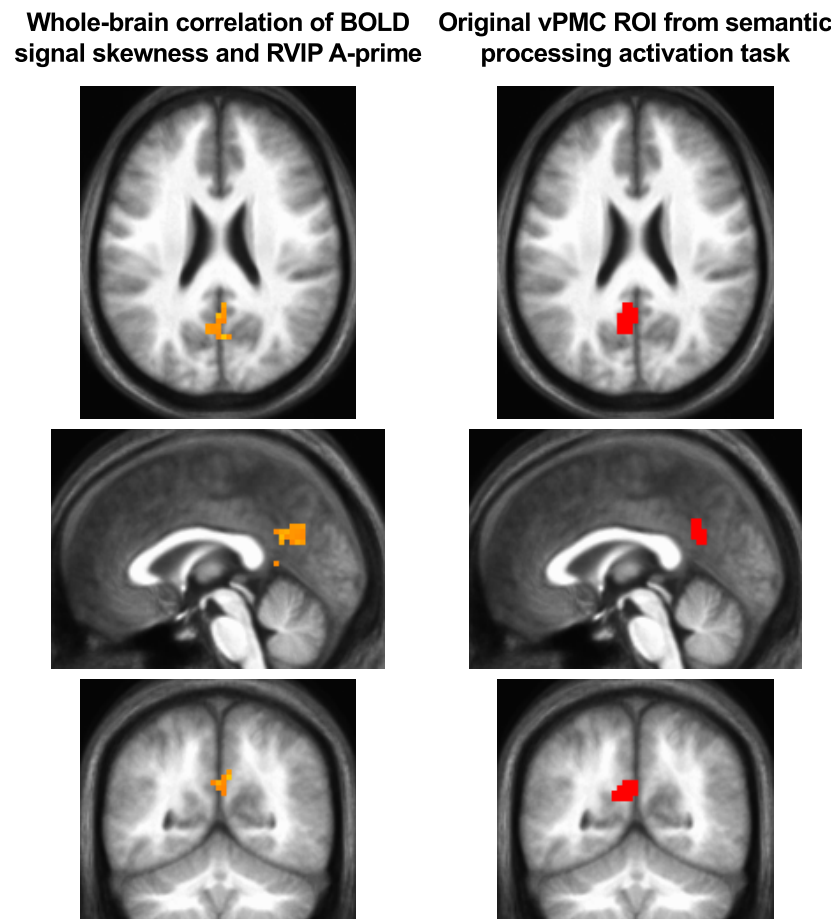

Figure 2. Comparison of the locations of the largest cluster from the whole-brain correlation analysis of voxelwise BOLD signal skewness and individual RVIP $A^{\prime}$ scores surviving an exploratory threshold of $p<0.05$ uncorrected (left), and of the vPMC ROl from the lexical decision task (right). The underlay image is the average of all the individual MNI-normalized T1-weighted volumes and the sections are cut on the coordinates of the center-of-mass of the cluster from the whole-brain correlation analysis of RVIP $A^{\prime}$ and BOLD signal skewness.

(Barnes et al., 2009) from the former could, in principle, have affected brain activity recorded during the latter. In particular, subjects who exhibited a strong vPMC activation for conceptual processing during the lexical-decision task (contrast: word vs nonword) could show a tendency for the $\mathrm{VPMC}$ to remain activated, and thus a more negative/less positive skewness of the $\mathrm{VPMC}$ BOLD signal, during the attention-to-breathing task. This possibility was assessed by a Pearson's correlation test between individual vPMC BOLD percentage signal changes for the word-versusnonword contrast in the lexical-decision task and vPMC BOLD signal skewness values during attention-to-breathing.

Functional connectivity analysis. To examine individual and group differences in the spatial structure of the DMN, we performed an fc analysis using the whole-brain EPI data, preprocessed and filtered as described above, and the vPMC ROI as the reference seed. Each subject's brain map of Pearson's correlation coefficients with the $\mathrm{vPMC}$ seed was converted to a $z$-score map via Fisher's transformation $\left(z=\tan h^{-1}(r)=\frac{1}{2} \ln \frac{1+r}{1-r}\right)$ to improve distributional characteristics. The individual $z$-score maps were then entered into the following second-level analyses to test for group effects. Separate 

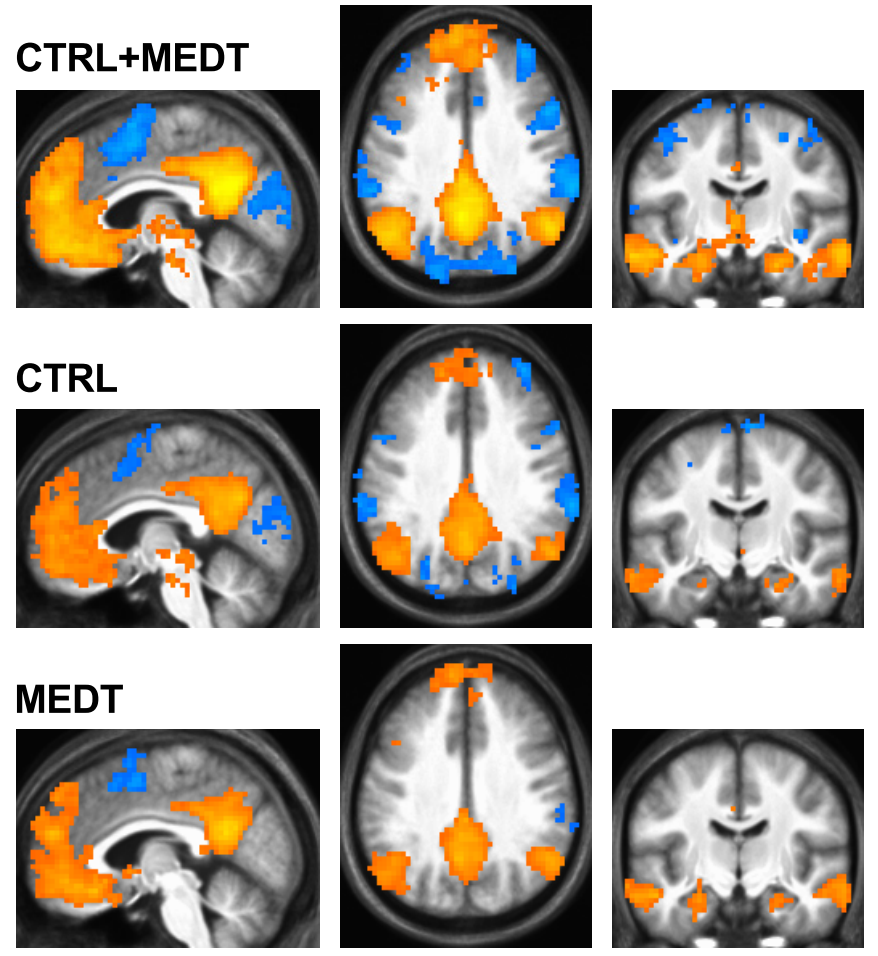

$x=4$

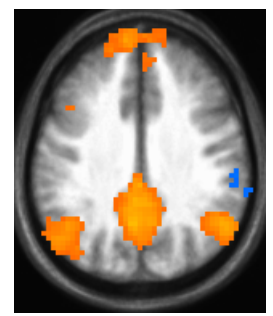

$z=35$

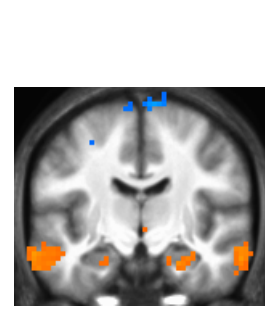

$\mathbf{z}$
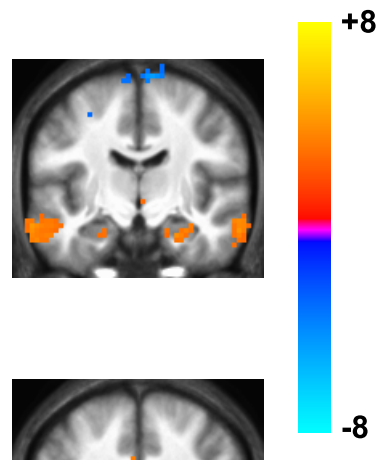

Figure 3. Z-scores statistical maps of functional connectivity with the vPMC seed at a corrected threshold of $\alpha<0.05$, for the pooled CTRL + MEDT groups (top row), the CTRL group (middle row), and the MEDT group (bottom row). The fc maps are overlayed on the average of the individual T1-weighted images, and the three orthogonal views, at MNI coordinates $(4,-16,35)$, have been chosen to illustrate both the cortical and the hippocampal components of the DMN.

Table 2. Significant clusters for the regression analysis of RVIP $A^{\prime}$ scores onto the vPMC-fc maps

\begin{tabular}{llllll}
\hline & & \multicolumn{3}{l}{ MNI coordinates } \\
\cline { 4 - 5 } Region & Size (in voxels) & z-value & $x$ & $y$ & $z$ \\
\hline RIFG & 38 & +4.15 & +39 & +30 & +12 \\
R TPJ & 36 & +4.20 & +63 & -45 & +27 \\
\hline
\end{tabular}

R, right.

one-sample $t$ tests were conducted for the CTRL group, the MEDT group, and the composite CTRL + MEDT group, in addition to a two-sample $t$ test comparing directly the two groups (CTRL - MEDT). Furthermore, a second-level regression analysis was performed testing for a linear relationship between the individual vPMC- $f c$ maps and the RVIP $A^{\prime}$ scores. All the resulting group-level statistical maps were thresholded at the combined single-voxel significance level of $p<0.005$ and cluster size $k \geq$ 32 voxels; these values were determined by the AFNI routine $3 \mathrm{dClustSim}$ via a Monte Carlo simulation of the cluster size distribution under the null hypothesis (Forman et al., 1995), to yield a family-wise error rate of $\alpha<0.05$.

To assess directly the role of the global GM signal on the observed pattern of correlations between the vPMC-fc maps and the RVIP $A^{\prime}$ scores, we performed a partial correlation analysis of the BOLD time courses of the selected regions (Marrelec et al., 2006; Smith et al., 2011), including the GM average BOLD time course as a virtual additional ROI. The average time courses from all the ROIs (GM ROI included) were extracted from the EPI time series, corrected for slice-timing and head movement, and orthogonalized to the nuisance variates of WMe signal, LVe signal, and motion parameters but, crucially, not to the GM signal. The estimated partial correlation coefficients, after being Fishertransformed, were entered into a pooled group $t$ test for difference against zero. Since partial correlation assesses the linear relationship between two variables when concomitant correlations with other variables are discounted, this procedure allowed us to examine the specific correlations between the time courses of selected ROIs vis-à-vis their correlation with the global GM signal.

\section{Results \\ Group differences in vPMC BOLD \\ signal skewness}

The distribution of the vPMC BOLD signal skewness values in the two experimental groups is shown in Figure $1 B$, with a median of -0.17 for control subjects and +0.11 for meditators.

A Wilcoxon rank-sum test for the a priori hypothesis of a positive MEDT CTRL difference in the vPMC BOLD signal skewness was significant at $p=0.0050$; the same test performed with the omission of the three outlier subjects (Fig. $1 B$, circles) yielded a significance value of $p=$ 0.000085 (median values: CTRL $=-0.21$, MEDT $=+0.11)$. The test performed on EPI data preprocessed without GM regression was not significant $(p=0.22)$.

None of the other regions of the DMN activated by conceptual processing in the lexical-decision task displayed a $p$ value $<0.05$ for the same test. Furthermore, the individual vPMC signal changes for the word-versus-nonword contrast in the lexical-decision task were not significantly associated with the vPMC BOLD skewness values during the attention-tobreathing run (Pearson's $r=0.28, p=0.19$ ), providing no substantial evidence of hysteresis effects from the previous task on the vPMC BOLD skewness. Finally, the analysis of the available respiration and heart rate data did not reveal any significant difference between MEDT and CTRL or correlation with the vPMC BOLD signal skewness (all $p>0.05$ ), and no subject was observed via the eye-tracking camera falling patently asleep during the scan.

\section{Correlation between vPMC BOLD skewness and RVIP performance}

Individual scores in the RVIP task ( $A^{\prime}$ and RT) were significantly correlated with the vPMC BOLD skewness values during the attention-to-breathing condition across all subjects (Fig. 1C,D). In accordance with the experiment's hypotheses, subjects displaying a more negative (or less positive) vPMC signal skewness were both less accurate $\left(A^{\prime}\right.$ : Pearson's $r=0.53$, one-tailed $p=$ 0.0039 ) and slower (RT: $r=-0.53$, one-tailed $p=0.0039)$ in their response to the RVIP target stimuli. There was no evidence for a significant difference in the above correlations for CTRL and MEDT as assessed by the interaction term of skewness and subject group in an ANCOVA model on the RVIP scores with separate slopes for each subject group $\left(A^{\prime}: p=0.29\right.$, RT: $\left.p=0.43\right)$. Furthermore, the correlations of vPMC BOLD skewness and RVIP scores were still significant when performed on EPI data preprocessed without GM regression, albeit less so $\left(A^{\prime}\right.$ : Pearson's $r=0.44$, one-tailed $p=0.016$; RT: $r=-0.50$, one-tailed $p=$ 0.006). None of the other regions of the DMN activated by the lexical-decision task displayed a correlation with RVIP scores 
that survived the Bonferroni-corrected ( 8 ROIs) threshold of $p=0.05 / 8=0.0063$.

The whole-brain voxelwise regression of individual RVIP $A^{\prime}$ scores onto the BOLD signal skewness yielded a statistical parametric map that was quite noisy. It is nonetheless interesting to note that, at the exploratory threshold of single-voxel $p<$ 0.05 (uncorrected), the largest suprathreshold cluster (center-of-mass MNI coordinates: $0,-57,23$; cluster size $=79$ voxels) was located in the same anatomical region of the original vPMC ROI (Fig. 2).

\section{vPMC-seeded functional connectivity} The $\mathrm{fc}$ analysis with the vPMC seed resulted in very similar statistical maps for the CTRL and MEDT groups (Fig. 3). Indeed, when testing directly for group differences, no clusters survived the whole-brain corrected threshold of $\alpha<$ 0.05 (see Materials and Methods, above).

The whole-brain regression of individual RVIP $A^{\prime}$ scores onto the vPMC-fc maps yielded two significant clusters, one in the inferior frontal gyrus (IFG) and the other in the temporoparietal junction (TPJ), both in the right hemisphere (Table 2; Fig. 4, top).

To examine such findings in closer detail and to assess the contribution of the global GM signal to the observed pattern of functional connectivity, a partial correlation analysis was performed with the average BOLD time courses of three ROIs of interest (vPMC, right IFG, right TPJ), plus a virtual global GM ROI (see Materials and Methods, above). The results showed a strong positive partial correlation of all ROIs' time courses with the GM signal and a negative partial correlation of vPMC and right TPJ, while the remaining partial correlations failed to reach significance across subjects (Fig. 4, bottom left). Finally, the vPMC-right TPJ (Fisher-transformed) partial correlation coefficients were plotted against the individual RVIP $A^{\prime}$ scores (Fig. 4, bottom right), revealing that subjects with negative partial correlations performed more poorly in the RVIP task compared with subjects with partial correlation values close to zero or positive.

\section{Discussion}

A number of studies have linked DMN activity to the spontaneous occurrence of task-unrelated thoughts, a process often competing with goal-directed attention (Sonuga-Barke and Castellanos, 2007). We analyzed the BOLD signal from a key DMN node, the vPMC, collected during a self-regulated attention-to-breathing task in habitual Zen meditators, whose practice entails an active familiarity with attentional regulation in the face of mind-wandering, and matched controls. Using the skewness of the BOLD time course from the vPMC as an estimate of the relative incidence of vPMC high- and low-activity states, we observed a significant group difference for this measure, in line with the hypothesis of a regulatory effect of meditative practice on DMN spontaneous activity.

We also observed a significant association, across all subjects, between the vPMC BOLD signal skewness and attentional pertion is drawn for reference.
R IFG

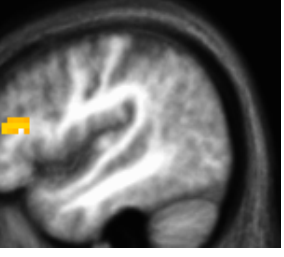

GM

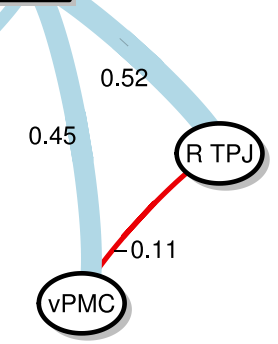

R TPJ
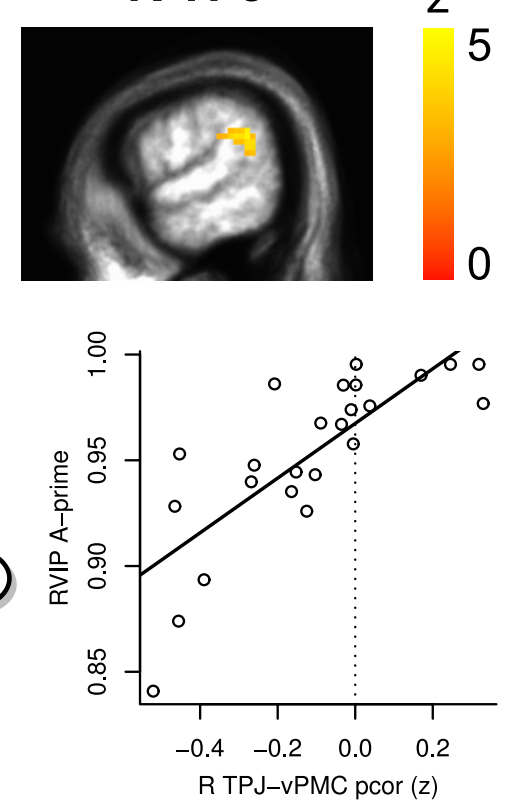

4. Top, Significant clusters for the correlation analysis of the VPMC-fc maps with the individual RVIP $A^{\prime}$ scores. Bottom (

formance in the RVIP task. The vPMC is characterized by important connections with hippocampal and parahippocampal cortices (Vogt and Pandya, 1987; Kobayashi and Amaral, 2003; Vincent et al., 2006; Vogt et al., 2006; Kahn et al., 2008; Greicius et al., 2009; Margulies et al., 2009; Leech et al., 2011) and is activated by both visuospatial navigation (Maguire, 2001; Vogt et al., 2004) and episodic memory retrieval (Andreasen et al., 1995; Burianova and Grady, 2007), functions that have been proposed to originate from a common hippocampal mechanism of navigation through both physical and mnemonic space mediated by theta oscillations (Buzsáki, 2005). More specifically, the organized activation of vPMC and hippocampal cortices has been implicated in orienting attention to internally generated representations (Wagner et al., 2005; Huijbers et al., 2011). In this perspective, the vPMC BOLD signal skewness, by providing a measure of the relative incidence of high- and low-activity states in the vPMC over time, may index the likelihood of such spontaneous internally generated representations to access consciousness and thereby interfere with externally directed attention.

The results of the fc analysis provide further insight into the relationship between attentional control and vPMC activity. According to an influential theory (Corbetta and Shulman, 2002), TPJ and IFG are the main nodes of a right-hemisphere ventral attention network (VAN) involved in stimulus-driven attentional reorienting. More specifically, TPJ is activated by behaviorally relevant stimuli and deactivates when irrelevant information needs to be filtered out (Corbetta et al., 2008). Notably, there is evidence for complementary roles of VAN and DMN regarding the directionality of awareness. Prestimulus activity in right IFG and TPJ has been shown to correlate positively with conscious perception of a somatosensory external stimulus, 
while a correlation of the opposite sign was observed for the DMN (Boly et al., 2007). In a recent resting state study using random phenomenological sampling, right TPJ and IFG activities were positively associated with the self-reported intensity of externally directed awareness, while DMN activity correlated with the intensity of internally directed awareness (Vanhaudenhuyse et al., 2011). Also, TPJ has been shown to be involved in attentional recovery after a momentary lapse, which was itself associated with a reduced deactivation of DMN areas (Weissman et al., 2006).

In the present study, we observed that subjects displaying a strong negative partial correlation between TPJ and vPMC performed more poorly in the RVIP task (Fig. 4, bottom right). If, with the caveats expressed in Materials and Methods about the still poorly understood meaning of the global signal, a negative partial correlation between two regions can be interpreted as functional competition above and beyond their shared variance with the whole GM, then subjects exhibiting a marked negative vPMC-TPJ partial correlation may face an increased likelihood of transient phases of inattentional blindness to external events (Todd et al., 2005) when the vPMC spontaneously activates, which will in turn be reflected in poorer RVIP scores. Conversely, a vPMC-TPJ partial correlation approaching zero or reaching positive values during attention to breathing may indicate that the VAN activity is less prone to be inhibited by spontaneous instances of DMN activation (Corbetta et al., 2008; Anticevic et al., 2010), so that target stimuli are better detected when engaging in the RVIP task. Note that, in this perspective, there are at least two potential cognitive strategies for reaching a high score in the RVIP task in the presence of spontaneous, internally generated distractors: suppress task-unrelated thoughts by inhibiting DMN activity, or be able to effectively activate the VAN independently of (or perhaps even in weak synergy with) the DMN activity state, so that behaviorally relevant external stimuli can be detected despite the occurrence of task-unrelated thoughts. The present findings seem to favor the latter interpretation. Furthermore, the vPMC BOLD signal skewness and the vPMC-TPJ fc may reflect distinct, but not mutually exclusive, mechanisms capable of influencing externally directed attention: on the one hand, the vPMC signal skewness provides an estimate of the abundance of high-activity states in the vPMC over time and, therefore, of the potential for spontaneous internally generated representations to interfere with externally directed attention by gaining access to consciousness; on the other hand, such internally generated material may be able to affect significantly the detection of external stimuli only when coupled with a transient deactivation of the TPJ, a situation more likely to occur in subjects exhibiting a strong negative vPMC-TPJ functional connectivity.

An important feature of the present results is that fMRI data collected during attention to breathing were statistically associated with RVIP scores measured on a different day. While this could arguably be seen as a limitation of the study, potentially weakening the link between fMRI and behavioral data by illcontrolled time effects, it can also suggest that the observed associations are more likely to reflect trait-like individual differences regarding attentional function, rather than state contingencies (e.g., unspecific arousal effects). The tendency to mind-wander, in particular, has been shown to be a fairly stable cognitive characteristic across time, daily activities, and contexts (McVay et al., 2009), and several studies have already demonstrated remarkable associations between neuroimaging data collected under stimulus-free resting state paradigms and individual behavioral performance, psychological traits, and/or brain activa- tion during a task (Hampson et al., 2006; Seeley et al., 2007; Kelly et al., 2008; Di Martino et al., 2009; Cox et al., 2010; Bonnelle et al., 2011). In this perspective, and if the present results are confirmed with larger samples, the vPMC BOLD signal skewness and the vPMC-TPJ partial correlations values may provide a useful endophenotype of attention capability in healthy individuals as well as in clinical conditions such as ADHD (Tian et al., 2006; Sonuga-Barke and Castellanos, 2007; Castellanos et al., 2008). Given the recent evidence linking the DMN and Alzheimer's disease, with a potential connection between the DMN metabolic rate and amyloid deposition (Buckner et al., 2005, 2009), it may be also worthwhile to explore how the skewness measure proposed here relates to the early, as well as to the more advanced, stages of the disease.

\section{Caveats and limitations of the study}

A number of caveats and unresolved questions need to be underscored. First, while the observed data support the hypothesis of an effect of meditative practice on the skewness of the vPMC BOLD signal (MEDT > CTRL), selection and confounding biases cannot be ruled out due to the case-control experimental design; also, the limited number of subjects within each group suggests caution in generalizing the group comparison results until confirmed by larger sample sizes. Second, the impact of the attention-to-breathing instructions versus the more common unconstrained resting state protocols on the reported findings cannot be assessed in this study, an issue we intend to clarify in future investigations; likewise, it will be important in future studies to monitor more precisely the attentional and arousal state of the subjects via, e.g., quantitative eye-tracking and pupillometry, a measure we were unfortunately unable to obtain due to technical issues. Finally, the observed results are contingent on, or are enhanced by, the use of GM regression during data preprocessing: as noted in Methods and Materials, the roles of global versus local BOLD fluctuations in contributing to specific patterns of intrinsic activity, as well as their functional import, are not well understood yet; further research in this area, ideally combining electrophysiological and fMRI recordings, holds promises to crucially affect our comprehension of the resting state and brain function in general.

\section{References}

Andreasen NC, O'Leary DS, Cizadlo T, Arndt S, Rezai K, Watkins GL, Ponto LL, Hichwa RD (1995) Remembering the past: two facets of episodic memory explored with positron emission tomography. Am J Psychiatry 152:1576-1585.

Anticevic A, Repovs G, Shulman GL, Barch DM (2010) When less is more: TPJ and default network deactivation during encoding predicts working memory performance. Neuroimage 49:2638-2648.

Ashburner J, Friston KJ (2005) Unified segmentation. Neuroimage 26:839-851.

Barnes A, Bullmore ET, Suckling J (2009) Endogenous human brain dynamics recover slowly following cognitive effort. PLoS One 4:e6626.

Binder JR, Frost JA, Hammeke TA, Bellgowan PS, Rao SM, Cox RW (1999) Concep-tual processing during the conscious resting state: a functional MRI study. J Cogn Neurosci 11:80-95.

Binder JR, Swanson SJ, Hammeke TA, Sabsevitz DS (2008) A comparison of five fMRI protocols for mapping speech comprehension systems. Epilepsia 49:1980-1997.

Birn RM, Diamond JB, Smith MA, Bandettini PA (2006) Separating respiratory-variation-related fluctuations from neuronal-activity-related fluctuations in fMRI. Neuroimage 31:1536-1548.

Birn RM, Smith MA, Jones TB, Bandettini PA (2008) The respiration response function: the temporal dynamics of fMRI signal fluctuations related to changes in respiration. Neuroimage 40:644-654.

Boly M, Balteau E, Schnakers C, Degueldre C, Moonen G, Luxen A, Phillips C, 
Peigneux P, Maquet P, Laureys S (2007) Baseline brain activity fluctuations predict somatosensory perception in humans. Proc Natl Acad Sci U S A 104:12187-12192.

Bonnelle V, Leech R, Kinnunen KM, Ham TE, Beckmann CF, De Boissezon X, Greenwood RJ, Sharp DJ (2011) Default mode network connectivity predicts sustained attention deficits after traumatic brain injury. J Neurosci 31:13442-13451.

Buckner RL, Carroll DC (2007) Self-projection and the brain. Trends Cogn Sci 11:49-57.

Buckner RL, Snyder AZ, Shannon BJ, LaRossa G, Sachs R, Fotenos AF, Sheline YI, Klunk WE, Mathis CA, Morris JC, Mintun MA (2005) Molecular, structural, and functional characterization of Alzheimer's disease: evidence for a relationship between default activity, amyloid, and memory. J Neurosci 25:7709-7717.

Buckner RL, Sepulcre J, Talukdar T, Krienen FM, Liu H, Hedden T, AndrewsHanna JR, Sperling RA, Johnson KA (2009) Cortical hubs revealed by intrinsic functional connectivity: mapping, assessment of stability, and relation to Alzheimer's disease. J Neurosci 29:1860-1873.

Burianova H, Grady CL (2007) Common and unique neural activations in autobiographical, episodic, and semantic retrieval. J Cogn Neurosci 19:1520-1534.

Buzsáki G (2005) Theta rhythm of navigation: link between path integration and landmark navigation, episodic and semantic memory. Hippocampus 15:827-840.

Castellanos FX, Margulies DS, Kelly C, Uddin LQ, Ghaffari M, Kirsch A, Shaw D, Shehzad Z, Di Martino A, Biswal B, Sonuga-Barke EJ, Rotrosen J, Adler LA, Milham MP (2008) Cingulate-precuneus interactions: a new locus of dysfunction in adult attention-deficit/hyperactivity disorder. Biol Psychiatry 63:332-337.

Chang C, Glover GH (2009) Effects of model-based physiological noise correction on default mode network anti-correlations and correlations. Neuroimage 47:1448-1459.

Christoff K, Ream JM, Gabrieli JD (2004) Neural basis of spontaneous thought processes. Cortex 40:623-630.

Christoff K, Gordon AM, Smallwood J, Smith R, Schooler JW (2009) Experience sampling during fMRI reveals default network and executive system contributions to mind wandering. Proc Natl Acad Sci U S A 106:8719-8724

Corbetta M, Shulman GL (2002) Control of goal-directed and stimulusdriven attention in the brain. Nat Rev Neurosci 3:201-215.

Corbetta M, Patel G, Shulman GL (2008) The reorienting system of the human brain: from environment to theory of mind. Neuron 58:306-324.

Cox CL, Gotimer K, Roy AK, Castellanos FX, Milham MP, Kelly C (2010) Your resting brain cares about your risky behavior. PLoS One 5:e12296.

Cox RW (1996) AFNI: software for analysis and visualization of functional magnetic resonance neuroimages. Comput Biomed Res 29:162-173.

Di Martino A, Shehzad Z, Kelly C, Roy AK, Gee DG, Uddin LQ, Gotimer K, Klein DF, Castellanos FX, Milham MP (2009) Relationship between cingulo-insular functional connectivity and autistic traits in neurotypical adults. Am J Psychiatry 166:891-899.

Forman SD, Cohen JD, Fitzgerald M, Eddy WF, Mintun MA, Noll DC (1995) Improved assessment of significant activation in functional magnetic resonance imaging (fMRI): use of a cluster-size threshold. Magn Reson Med 33:636-647.

Fox MD, Zhang D, Snyder AZ, Raichle ME (2009) The global signal and observed anticorrelated resting state brain networks. J Neurophysiol 101:3270-3283.

Fransson P, Marrelec G (2008) The precuneus/posterior cingulate cortex plays a pivotal role in the default mode network: evidence from a partial correlation network analysis. Neuroimage 42:1178-1184.

Green DM, Swets JA (1966) Signal detection theory and psychophysics. New York: Wiley.

Greicius MD, Krasnow B, Reiss AL, Menon V (2003) Functional connectivity in the resting brain: a network analysis of the default mode hypothesis. Proc Natl Acad Sci U S A 100:253-258.

Greicius MD, Supekar K, Menon V, Dougherty RF (2009) Resting-state functional connectivity reflects structural connectivity in the default mode network. Cereb Cortex 19:72-78.

Hagmann P, Cammoun L, Gigandet X, Meuli R, Honey CJ, Wedeen VJ, Sporns O (2008) Mapping the structural core of human cerebral cortex. PLoS Biology 6:e159.

Hampson M, Driesen NR, Skudlarski P, Gore JC, Constable RT (2006)
Brain connectivity related to working memory performance. J Neurosci 26:13338-13343.

Hassabis D, Maguire EA (2007) Deconstructing episodic memory with construction. Trends Cogn Sci 11:299-306.

Huijbers W, Pennartz CM, Cabeza R, Daselaar SM (2011) The hippocampus is coupled with the default network during memory retrieval but not during memory encoding. PLoS One 6:e17463.

Jo HJ, Saad ZS, Simmons WK, Milbury LA, Cox RW (2010) Mapping sources of correlation in resting state FMRI, with artifact detection and removal. Neuroimage 52:571-582.

Joanes DN, Gill CA (1998) Comparing measures of sample skewness and kurtosis. J R Stat Soc Series D Statistic 47:183-189.

Kahn I, Andrews-Hanna JR, Vincent JL, Snyder AZ, Buckner RL (2008) Distinct cortical anatomy linked to subregions of the medial temporal lobe revealed by intrinsic functional connectivity. J Neurophysiol 100:129-139.

Kelly AM, Uddin LQ, Biswal BB, Castellanos FX, Milham MP (2008) Competition between functional brain networks mediates behavioral variability. Neuroimage 39:527-537.

Kobayashi Y, Amaral DG (2003) Macaque monkey retrosplenial cortex. II. Cortical afferents. J Comp Neurol 466:48-79.

Leech R, Kamourieh S, Beckmann CF, Sharp DJ (2011) Fractionating the default mode network: distinct contributions of the ventral and dorsal posterior cingulate cortex to cognitive control. J Neurosci 31:3217-3224.

Lutz A, Slagter HA, Dunne JD, Davidson RJ (2008) Attention regulation and monitoring in meditation. Trends Cogn Sci 12:163-169.

Maguire EA (2001) The retrosplenial contribution to human navigation: a review of lesion and neuroimaging findings. Scand J Psychol 42:225-238.

Margulies DS, Vincent JL, Kelly C, Lohmann G, Uddin LQ, Biswal BB, Villringer A, Castellanos FX, Milham MP, Petrides M (2009) Precuneus shares intrinsic functional architecture in humans and monkeys. Proc Natl Acad Sci U S A 106:20069-20074.

Marrelec G, Krainik A, Duffau H, Pélégrini-Issac M, Lehéricy S, Doyon J, Benali H (2006) Partial correlation for functional brain interactivity investigation in functional MRI. Neuroimage 32:228-237.

Mason MF, Norton MI, Van Horn JD, Wegner DM, Grafton ST, Macrae CN (2007) Wandering minds: the default network and stimulus-independent thought. Science 315:393-395.

McKiernan KA, D'Angelo BR, Kaufman JN, Binder JR (2006) Interrupting the "stream of consciousness": an fMRI investigation. Neuroimage 29:1185-1191.

McVay JC, Kane MJ, Kwapil TR (2009) Tracking the train of thought from the laboratory into everyday life: an experience-sampling study of mind wandering across controlled and ecological contexts. Psychon Bull Rev 16:857-863.

Mizuno K (1996) Essentials of Buddhism. Tokyo: Kosei Publishing.

Murphy K, Birn RM, Handwerker DA, Jones TB, Bandettini PA (2009) The impact of global signal regression on resting state correlations: are anticorrelated networks introduced? Neuroimage 44:893-905.

Pagnoni G, Cekic M, Guo Y (2008) “Thinking about not-thinking”: neural correlates of conceptual processing during Zen meditation. PLoS One 3:e3083.

R Development Core Team (2011) R: a language and environment for statistical computing. Vienna: R Foundation for Statistical Computing.

Raichle ME, MacLeod AM, Snyder AZ, Powers WJ, Gusnard DA, Shulman GL (2001) A default mode of brain function. Proc Natl Acad Sci U S A 98:676-682.

Sahakian BJ, Owen AM (1992) Computerized assessment in neuropsychiatry using CANTAB: discussion paper. J R Soc Med 85:399-402.

Schölvinck ML, Maier A, Ye FQ, Duyn JH, Leopold DA (2010) Neural basis of global resting-state fMRI activity. Proc Natl Acad Sci USA 107:10238-10243.

Seeley WW, Menon V, Schatzberg AF, Keller J, Glover GH, Kenna H, Reiss AL, Greicius MD (2007) Dissociable intrinsic connectivity networks for salience processing and executive control. J Neurosci 27:2349-2356.

Shulman GL, Fiez JA, Corbetta M, Buckner RL, Miezin FM, Raichle ME, Petersen SE (1997) Common blood flow changes across visual tasks. II. Decreases in cerebral cortex. J Cogn Neurosci 9:648-663.

Smith SM, Miller KL, Salimi-Khorshidi G, Webster M, Beckmann CF, Nichols TE, Ramsey JD, Woolrich MW (2011) Network modelling methods for FMRI. Neuroimage 54:875-891.

Sonuga-Barke EJ, Castellanos FX (2007) Spontaneous attentional fluctua- 
tions in impaired states and pathological conditions: a neurobiological hypothesis. Neurosci Biobehav Rev 31:977-986.

Tian L, Jiang T, Wang Y, Zang Y, He Y, Liang M, Sui M, Cao Q, Hu S, Peng M, Zhuo Y (2006) Altered resting-state functional connectivity patterns of anterior cingulate cortex in adolescents with attention deficit hyperactivity disorder. Neurosci Lett 400:39-43.

Todd JJ, Fougnie D, Marois R (2005) Visual short-term memory load suppresses temporo-parietal junction activity and induces inattentional blindness. Psychol Sci 16:965-972.

Vanhaudenhuyse A, Demertzi A, Schabus M, Noirhomme Q, Bredart S, Boly M, Phillips C, Soddu A, Luxen A, Moonen G, Laureys S (2011) Two distinct neuronal networks mediate the awareness of environment and of self. J Cogn Neurosci 23:570-578.

Vincent JL, Snyder AZ, Fox MD, Shannon BJ, Andrews JR, Raichle ME, Buckner RL (2006) Coherent spontaneous activity identifies a hippocampal-parietal memory network. J Neurophysiol 96:3517-3531.

Vogt BA, Pandya DN (1987) Cingulate cortex of the rhesus monkey. II. Cortical afferents. J Comp Neurol 262:271-289.
Vogt BA, Hof PR, Vogt L (2004) Cingulate gyrus. In: The human nervous system, 2nd edition (Paxinos BA, Mai JK, eds.). pp 915-949. San Diego: Elsevier.

Vogt BA, Vogt L, Laureys S (2006) Cytology and functionally correlated circuits of human posterior cingulate areas. Neuroimage 29:452-466.

Wagner AD, Shannon BJ, Kahn I, Buckner RL (2005) Parietal lobe contributions to episodic memory retrieval. Trends Cogn Sci 9:445-453.

Weissenbacher A, Kasess C, Gerstl F, Lanzenberger R, Moser E, Windischberger C (2009) Correlations and anticorrelations in resting-state functional connectivity MRI: a quantitative comparison of preprocessing strategies. Neuroimage 47:1408-1416.

Weissman DH, Roberts KC, Visscher KM, Woldorff MG (2006) The neural bases of momentary lapses in attention. Nat Neurosci 9:971-978.

Wesnes K, Warburton DM (1983) Effects of smoking on rapid information processing performance. Neuropsychobiology 9:223-229.

Wirth M, Jann K, Dierks T, Federspiel A, Wiest R, Horn H (2011) Semantic memory involvement in the default mode network: a functional neuroimaging study using independent component analysis. Neuroimage 54: 3057-3066. 